Proceedings of the 1999 IEEE/ASME

International Conference on Advanced Intelligent Mechatronics

September 19-23, $1999 \cdot$ Atlanta, USA

\title{
Evolution of Urban Robotic System Developments
}

Hagen Schempf ${ }^{a}$, Edward Mutschler ${ }^{\mathrm{a}},{\text { Brian } \text { Chemel }^{\mathrm{a}} \text {, Shree Nayar }}^{\mathrm{a}}$, Colin Piepgras ${ }^{\mathrm{a}}$, William Crowley ${ }^{\mathrm{a}}$, Scott Boehmke $^{\mathrm{a}}$

a: Carnegie Mellon University Robotics Institute

5000 Forbes Ave.

Pittsburgh, PA 15213

hagen+@cmu.edu b: Columbia University

School of Computer Science

500 W., 120th Street

New York, NY 10027

nayar@cs.cmu.edu

\section{ABSTRACT}

Urban settings represent a challenging environment for teleoperated and autonomous robot systems. We present several different improved and novel robotic locomotion and exploration/inspection systems designed for the arenas of military reconnaissance and even entertainment/toys. The designs presented in this paper focus in on all- and flat-floor teleoperated./autonomous-capability robotic platforms developed at CMU over the past year. The Pandora and Minidora robot systems are tracked robot systems with self-contained computing, power and wireless communications systems. A sensor suite including stereoscopic and panospheric cameras, lightstripers and acoustic sonar-ring(s) allow the systems to operate autonomously. Individually, adjustable trackmodules give both of these robot systems extreme mobility in natural (vegetation, soils) and man-made (roads, steps) outdoor environments as well as indoor arenas (sewers, staircases, etc.). The Cyclops and Omniclops robot systems are self-driving spherical camera-platforms intended to be deployed on indoor flatfloor terrain for exploration tasks. Both systems also are fully self-contained in terms of locomotion, computing, power, and control. Both systems differ in their design for rolling and steering, as well as the camera sensor used - all these will be detailed in this paper. Locomotion for all the above systems was shown successfully over various flat and extreme terrains, including reconfiguration to best suit the terrain and enable future sensor-supported autonomous operations.

\section{INTRODUCTION}

Applications for such urban-terrain locomotion systems with mobile exploration/surveillance systems exists in several lines of work, including nuclear, entertainment/ toys, police or military domains. Remote surveillance systems remove the systems' operators from harm's way, giving them the opportunity to survey a potentially dangerous situation from a distance. Mobile robots with video transmission capabilities can also be used effectively as expendable "scouts" in military and disaster areas, giving advance information about pollution levels and other hazardous conditions - as a toy, these systems would represent a new dimension in entertainment not just for the young.

\section{OVERVIEW}

The system we will detail herein are shown in Figure 1, including Pandora, Minidora, Cyclops and Omniclops:
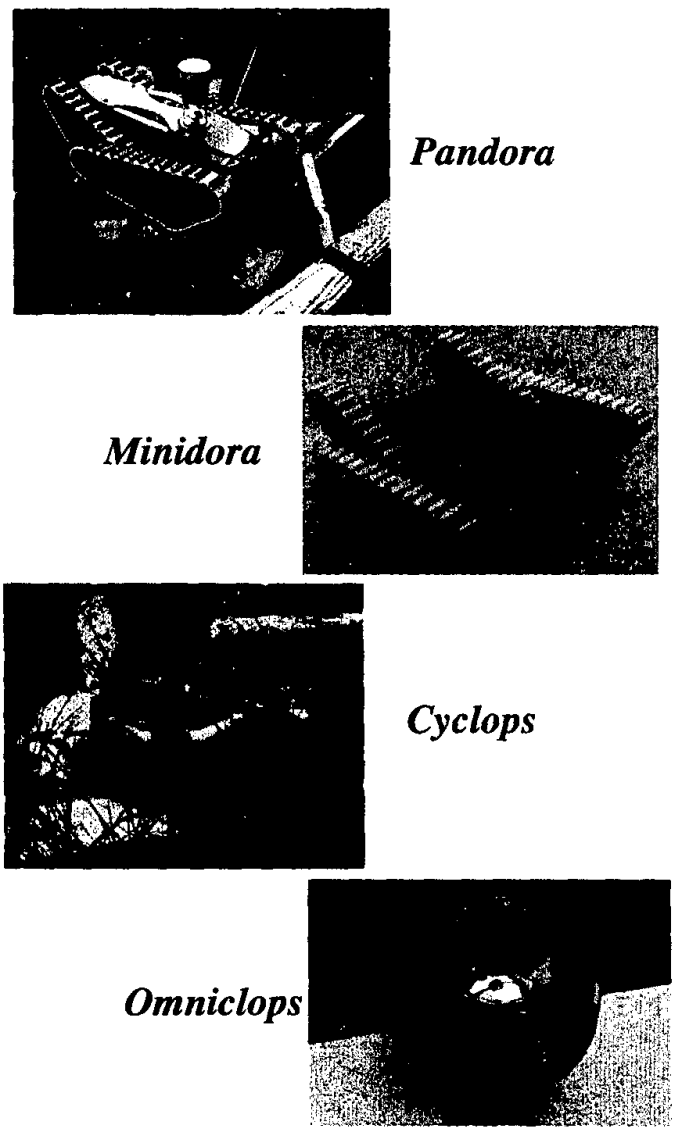

Figure I : Robot Suite Overview 


\section{Pandora \& Minidora - OUTDOOR ROUGH-TERRAIN SYSTEMS}

The Pandora system design developed for urban operations, consists of a reconfigurable dual tread-driven skid-steered robot vehicle (see Figure 2),

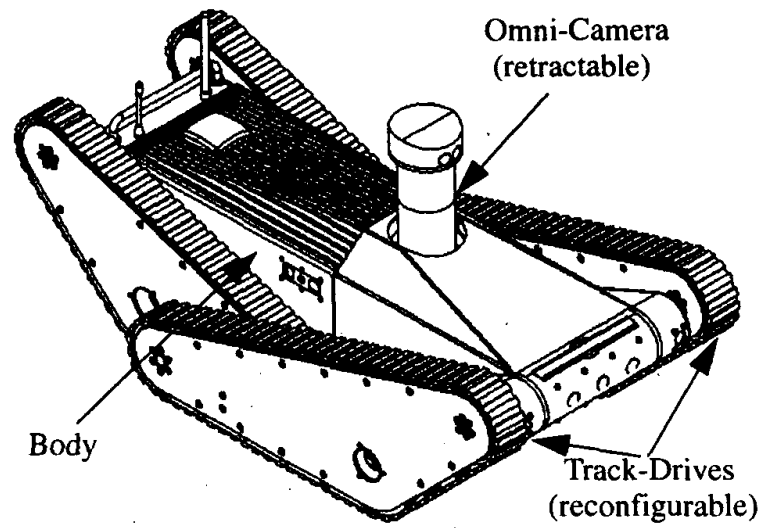

Figure 2 : CAD Rendering for prototype system

with specifications as detailed in the table below. The main systems within the Pandora vehicle system consist of the locomotor-modules (track-modules in this instantiation), the vehicle-chassis, the drivetrains, the electronics-enclosure, the omni-spherical camera, and the forward- and side-/rear-looking CCD-cameras and sonar systems (we consider Pandora a more compact, portable, integrated and autonomy-friendly relative of Remotec's ANDROS line [5], which itself is based on a patented german nuclear-response robot-design from the 80's).

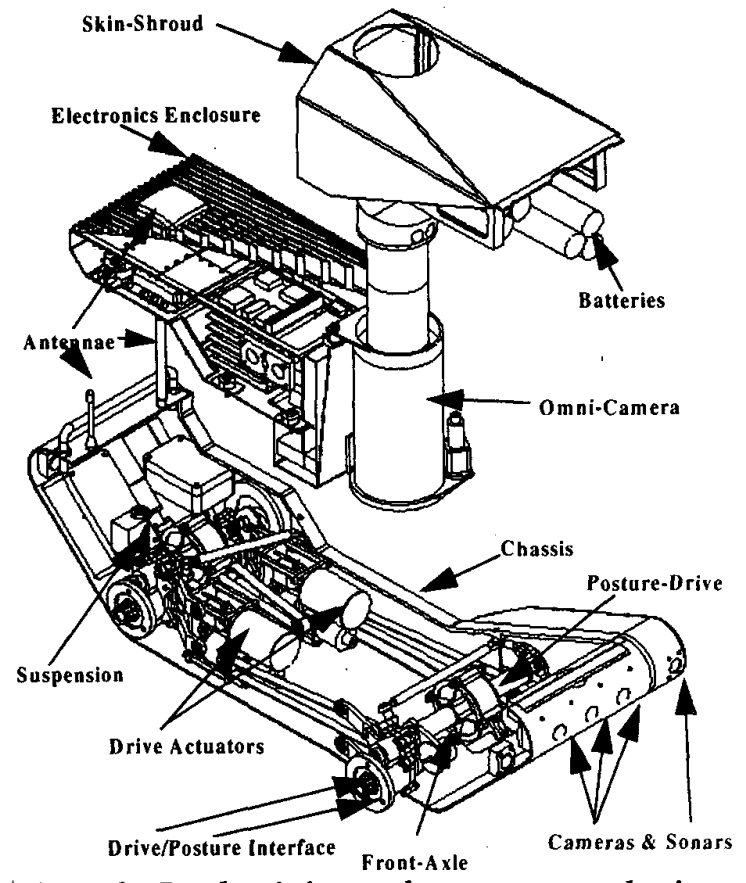

Figure 3 : Pandora's internal component packaging

The internal architecture and component packaging for the Pandora system are depicted in Figure 3. The Pandora system can orient its front- and rear pairs of tracks to allow it to climb up, -onto and -over a large variety of roads, curbs, bricks, boulders, steps, walls, ditches and through sewer-pipes (see Figure 4).

\begin{tabular}{|c|c|c|c|}
\hline \multirow[t]{2}{*}{ Speed } & Flat-/nncline-Driving & $5 \mathrm{mph}$ & 0 - 5 variable \\
\hline & Obstacle Climbing/Driving & $0.5 \mathrm{mph}$ & $0-0.5$ variable \\
\hline Weight & Overall & $65 \mathrm{lbs}$ & $+/ .5$ lbs. \\
\hline \multirow{4}{*}{$\begin{array}{l}\text { Size } \\
(L \times W \times H)\end{array}$} & Collapsed: & 33"x16"x11" & $+4 " H$ (w. camera) \\
\hline & Straddling: & 33"x16"x26"' & +4 "H (w. camera) \\
\hline & Ditch-Crossing: & 33"x16"x15" & +4 "H (w. camera) \\
\hline & Step-Climbing: & 46"x16"x11" & +4 "H (w. camera) \\
\hline Power & Li-Ion Sulphur-Dioxide Primaries & 36V Assy. & 1 hr. 1C Discharge \\
\hline \multirow{4}{*}{$\begin{array}{l}\text { Environment } \\
\text { Sensors }\end{array}$} & OmniCamera with integral window/motion SW & $360^{\circ} \mathrm{FOV}$ & $-5^{\circ}$ Down \\
\hline & Forward-looking Stereo CCD-Cameras & - & $\cdot$ \\
\hline & Filtered CCD camera with laser light-stripers & - & - \\
\hline & $4 \times 2$ vehicle-surrounding sonar-ring & $350 \mathrm{MHz}$ & multiplexed \\
\hline \multirow[t]{5}{*}{ Navigation Sensors } & DGPS Trimble receiver & - & - \\
\hline & Magnetic true-north Compass & $\cdot$ & - \\
\hline & Gravity-compensated Inclinometers & Pitch \& Roll & $+1.80^{\circ}$ \\
\hline & Solid-State Gyro & - & $+/-50 \%$ sec \\
\hline & Safety Switches: Mercury-bath binary camera-retract & $\cdot$ & - \\
\hline \multirow{2}{*}{ Communications } & $2.4 \mathrm{GHz}$ wireless $\mathrm{RF}$-modem & 9.6 KBaud & bi-directional \\
\hline & Switchable Video/Audio Transmitter & S-Band & transmit only \\
\hline
\end{tabular}



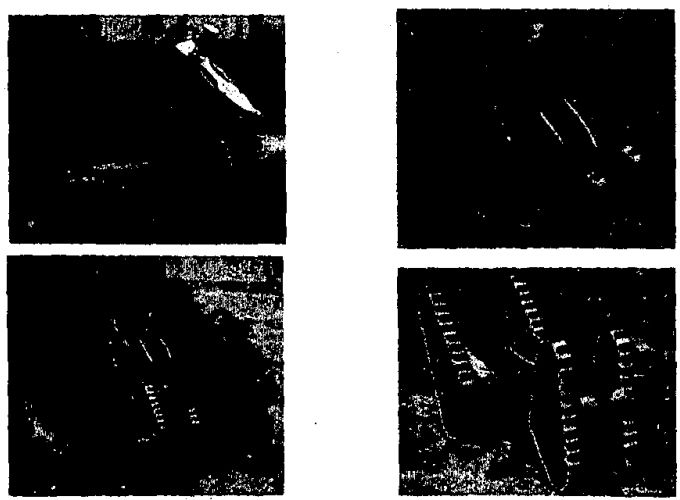

Figure 4 : Some of Pandora's possible configurations

The system is capable of reconfiguring its treads into an infinite number of configurations, with some of the more useful ones depicted in Figure 4 on a mock-up of the platform (16" wide, 35" long, 10" tall). The computing and power systems, as well as the derivation of the resulting speeds and climbing abilities for this system were detailed in [1].

The Minidora system (see Figure 5) is different from Pandora,

\section{Carbon-Fiber Body \\ (Cover \& Unibody}

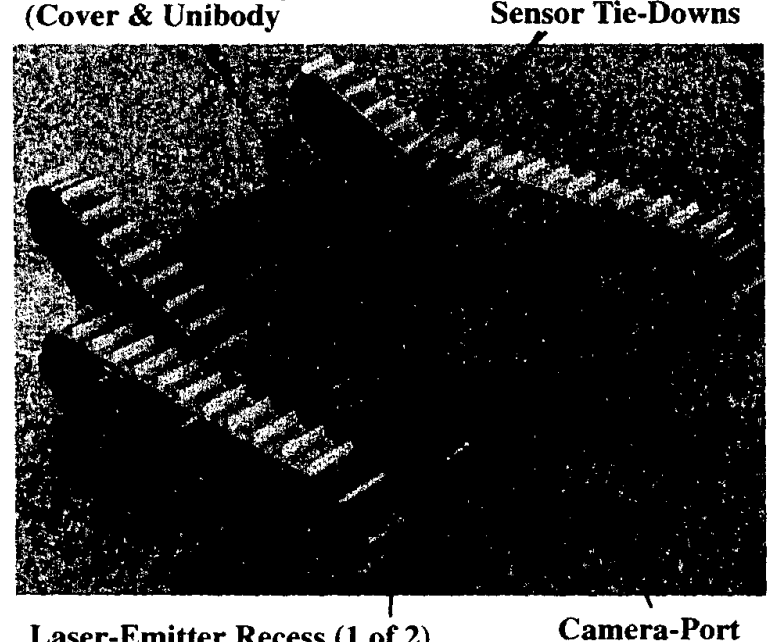

Laser-Emitter Recess (1 of 2)

Camera-Port

Figure 5 : Minidora prototype system

in that it represents a lighter, smaller and more teleoperated (rather than autonomy-capable) platform. Minidora (shown in Figure 6) is 16" x 18" x 7" in size $(\mathrm{L} \times \mathrm{W} \times \mathrm{H})$, and is made of laminated carbon-fiber reinforced resin-composite material, with embedded drive and posture systems. Internal battery and radio-frequency based electronic speed-control as are tied to a remote control-box (used by hobbyists) - this allows the system to be controlled remotely. An on-board forward-looking color mini-board camera provides the only visual feedback to the operator for making speed, heading and posture decisions. The system was built to allow it to be thrown from a multi-floor building, survive the fall and continue operation (akin to IS Robotics Inc. DARPAfunded URBI-platform) [6]. Notice that the system in its current baseline design does not include computing, as it is intended to be designed as a locomotion-chassis to be outfitted by the end-user.

The system however differs from others available commercially, in that it provides a substantial payload capacity and freedom in location of additional payload, by removable top- and bottom-covers, as well as strap-on rails on the top of the cover (albeit at the cost of not being able to flip itself over with an external payload attached to the top cover) - see Figure 6 for an inside-view of the packaging and lid-cover rails. The ability to readily add more payload, allows end-users to add mission-specific systems on-board, including sensors, computing, batteries, etc.

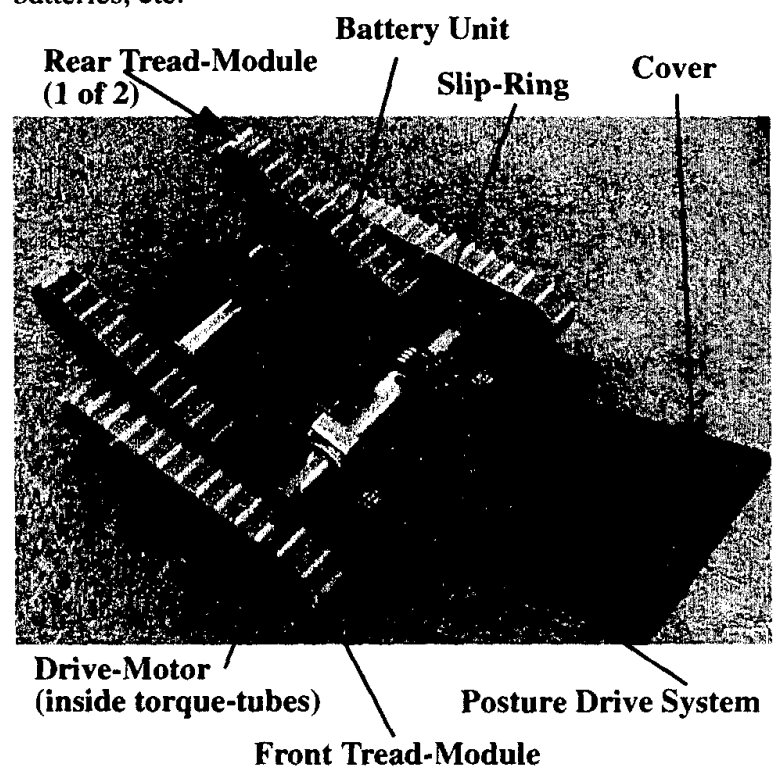

Figure 6 : Internal view of Minidora packaging

Minidora can flip itself over and take on any posture like Pandora. At a weight of less than 13 pounds, it can travel at 2 meters/sec. for over 3 hours, climb obstacles as high as 12 inches (conservatively), while providing rapid turning maneuvering capability $(\sim 300 \% \mathrm{sec}$.). Future expansion-space for on-board sensing and computing is available in the current design, and through simple cover remolding, additional protected-space can be readily generated. 


\section{Cyclops \& Omniclops - INDOOR SYSTEMS}

The Cyclops system, shown with its outer shell removed in Figure 7, consists of a roll-axis driven platform and an inertially-steered reaction-torque based heading-drive system.

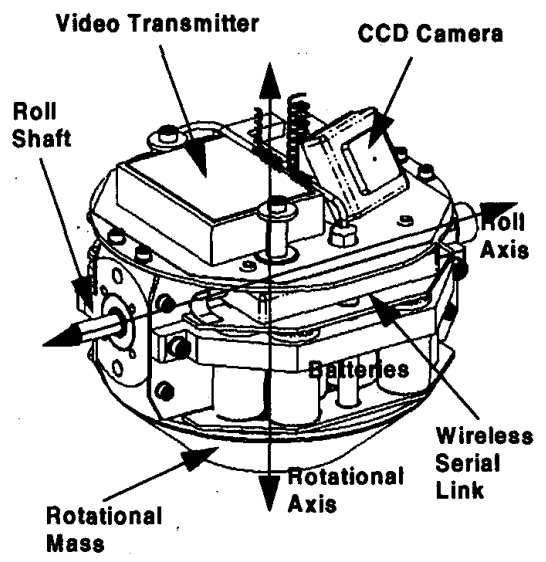

Figure 7 : A CAD rendering of the Cyclops prototype

On-board microprocessor and RF-based communications system, coupled with inclinometers and heading-compass, allowed simple off-board commands to control the system in the desired heading and speed. Video-feedback was provided by an on-board mini-board pinhole camera; the entire system was powered by $\mathrm{NiCad}$ power-cells (primaries). A depiction of the internal packaging and custom PC-boards is shown in Figure 8 - details on the computing-, power- and controller-architectures are presented in detail in [2]. The Omniclops system was a follow-on development between $\mathrm{CMU}$ and Columbia University, whereby they provided the optical system, and CMU developed the new locomotion and integrated design, prototyped, tested and demonstrated it.

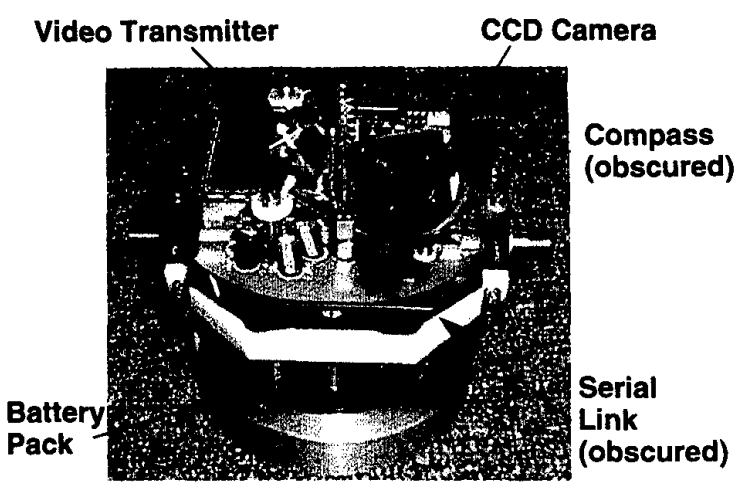

Figure 8 : Cyclops prototype sans external shell.

CMU developed both a passive (hand-thrown/-rolled) and an active (self-powered and driven/steered via remote control) version, both of which are shown in Figure 9:

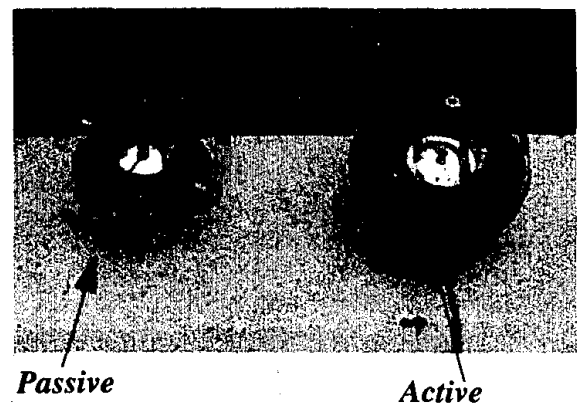

Figure 9 : Passive and active Omniclops systems

The systems weighed around 3 (passive) to 6 (active) pounds, and measured 8 inches in diameter - the reason for the large size was due to the cost and availability of optically-clear half-domes (smallest off-the-shelf we found were from Edmund Scientific). The passive

\begin{tabular}{|l|l|}
\hline \multicolumn{1}{|c|}{ COMPONENT } & \multicolumn{1}{c|}{ DESCRIPTION - Cyclops Only } \\
\hline \hline Robot Body Shell & 5.5 " diameter transparent acrylic sphere, with vertical tread strip \\
\hline Locomotion & 2-DOF mobility provided by forward/reverse roll mechanism with inertial steering \\
\hline Control & $\begin{array}{l}\text { Custom network of three embedded microcontrollers with } R F \text {-based serial link to operator con- } \\
\text { troller unit }\end{array}$ \\
\hline Navigation & Ceramic gyro, electronic compass linked to dead-reckoning microcontroller module \\
\hline Video Telemetry & CCD camera with RF video transmitter \\
\hline Power & Lithium-Ion primary battery pack, $\sim 1.5 \mathrm{hr}$ life for normal operation \\
\hline
\end{tabular}


Omniclops system is similar to Cyclops, in that it has a main horizontal gimbaled axis (unpowered in this case), while the heading of the unit is determined by the direction the operator 'rolls' it in a bowling-like motion. The innards of the active Omniclops system, shown in Figure 10,

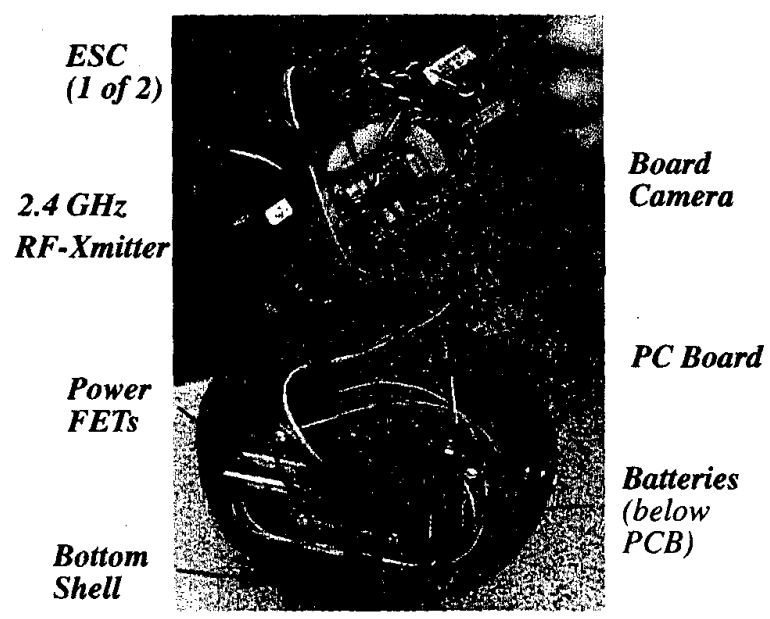

Figure 10 : Active Omniclops innards

illustrate the packaging of a simple RF-based (400 MHz) ESC-based driver system (used by hobbyists), and a highend video and audio transmitter $(2.4 \mathrm{GHz} @ 1$ Watt) for the video feedback from the camera. The drive-system for the active Omniclops, shown in Figure 11, consisted of a set of canted spheroidal cut-outs, which when driven independently would allow the system to roll in any direction based on a differential-drive/-steer principle. The above wheels have since been widened to provide a wider stance and thus a more stable motion (eliminates lateral oscillations). The batteries, rechargeable NiCads, are embedded at the bottom of the shell, to keep the center-ofgravity of the system as low as possible.

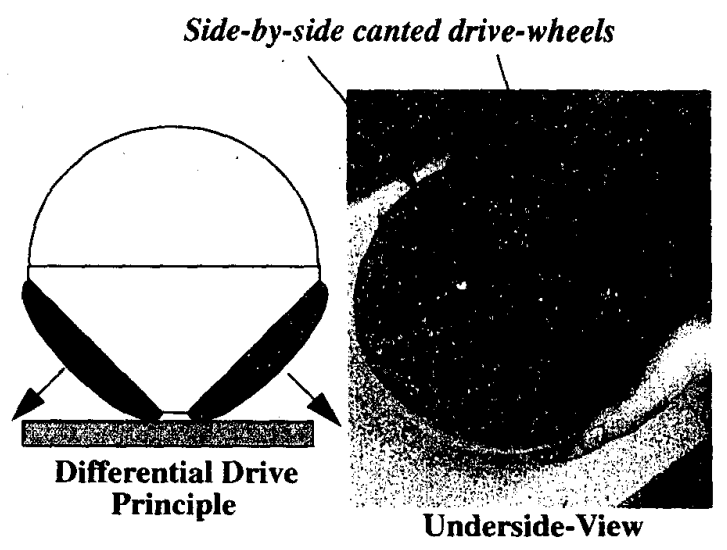

Figure 11 : Active Omniclops drive-wheel system
The mirror/lens and camera system used in this system provides real-time $360^{\circ}$ FOV imagery based on custom mirrors and optics developed by S. Nayar at Columbia [7], which when run through Columbia's dewarping software, generates real-time off-board de-warped images such as those shown in Figure 12,

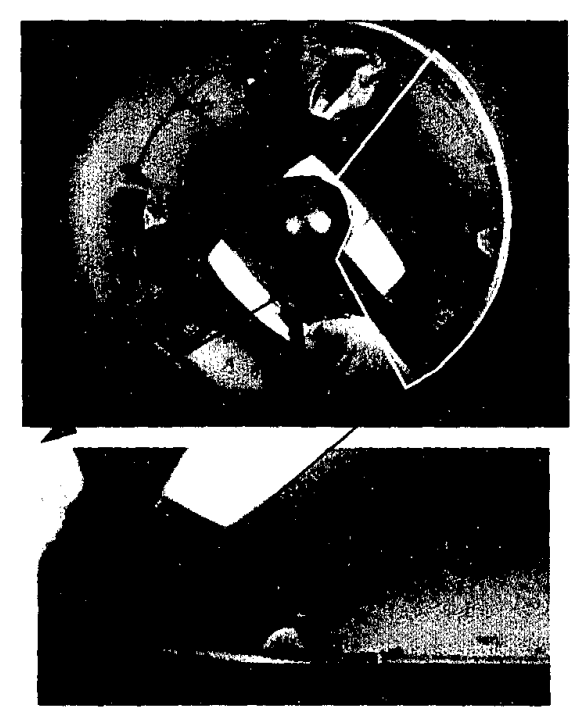

Figure 12 : Imagery from omnispheric camera

\section{SYSTEM PERFORMANCE EVALUATION}

Pandora and Minidora were evaluated in outdoor roughterrain scenarios and were found to substantially meet their claimed performance levels (see Figure 13),
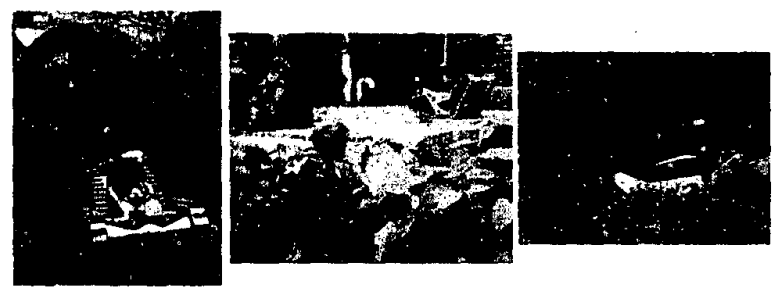

Figure 13 : Outdoor rough-terrain testing-scenarios

except for endurance - the endurance-levels were based on the use of costlier primary Lithium-ion batteries, rather than the readily-available and affordable Nickel-based batteries we used. From the myriad numbers of postures possible for Pandora and Minidora, it was determined that only about 5 are really essential and capable of handling $95 \%$ of all the terrains and obstacles we tested. The reduced weight and ease of transportability of the 
Minidora system proved to be major benefits in allowing mission/deployment flexibility. Omniclops was found to be far more maneuverable and capable using the new locomotion-system, as well as through the use of Columbia's omnispheric mirror/lens-camera system however the weight $(6 \mathrm{lbs}$.) and size ( 8 -inch dia.) of Omniclops will need to be reduced, allowing it to be readily portable in a pant-pocket and throwable akin to a baseball.

Seeing that user interfaces for teleoperated and even autonomous robot systems are the missing link in terms of robots really achieving their potential in the field, we decided to develop a fully-integrated, portable display, control \& computer interface for field robots (Figure 14):

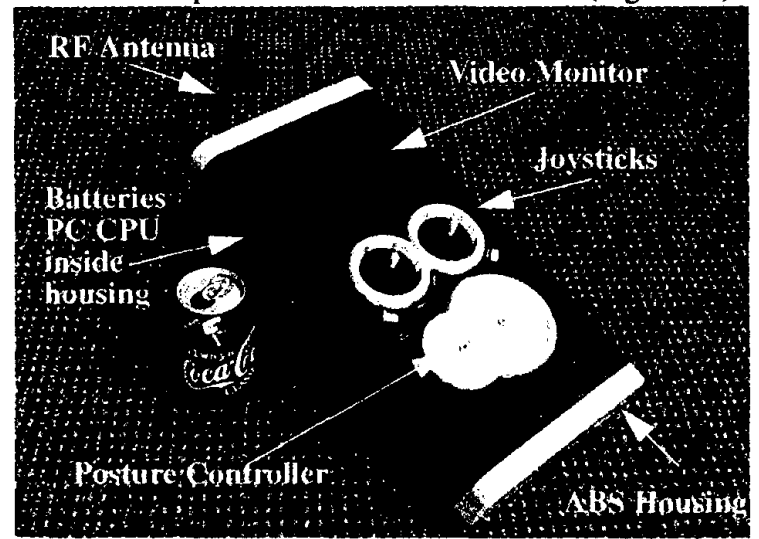

Figure 14 : Prototype portable display \& control system

The console worked very well, but will require further miniaturization and lightening before it can be considered a rugged portable controller for field-robot operations.

\section{FUTURE WORK}

Both CMU's and Columbia's ideas for improvements and novel systems will primarily focus on the use of currently available innovative materials (molded silicone-epoxy matrix-fibers for the body; optically-clear miniaturemolded PVCs for the dome) and the integration of ever improving power (smaller, lighter and denser), computing
(MIPS/volume or -weight) and communication (IR and/or RF-technology) systems - continued miniaturization is the primary goal! Both CMU and Columbia are committed to continue work in these areas over many years to come.

\section{ACKNOWLEDGEMENTS}

The Pandora and Cyclops work was funded by the Defense Advanced Research Project Agency (DARPA) under Contract № DAAL01-97-K-0165, in collaboration with Columbia University. The Omniclops work was funded by Columbia University as part of their MURI contract. The Minidora work was funded by discretionary funds of the PI. We wish to further acknowledge the contributions of Martial Hebert (sensing and autonomy), Chuck Thorpe (user interfaces and operations) and Anthony Stentz (planning) as well as other team-members for their valuable insight and contributions to the final system design. Patenting of Pandora, Minidora, Cyclops and Omniclops is pending.

\section{REFERENCES}

[1] Schempf et. al, "Pandora - Design of an Urban Robot System", IEEE R\&A Conference, Detroit, MI, May 13, 1999.

[2] Chemel, B. et al, "Cyclops - Miniature Robotic Reconnaissance System", Proc. of the IEEE R\&A Conference, Detroit, MI, May 14, 1999

[3] US Army Research Laboratories, "Pandora: A Robotic System for Operations in Urban Environments - Final Design Document", official contractreport submission, March 1998.

[4] Bekker, M.G., "Off-the-road Locomotion", University of Michigan Press, Ann Arbor, 1960

[5] REMOTEC, Inc. Product Literature, dated January 1, 1992

[6] IS Robotics Inc., "URBI WWW-page advertisement", http://www.isrobotics.com/projects/urban/ urban.html; Feb. '99.

[7] Nayar, S., Internal U. Columbia Omnicamera Design Document, not yet published, NY 1998 